\title{
BREATH HOLDING TIME AND OXYGEN SATURATION IN COVID AFFECTED NURSING STUDENTS-A COMPARATIVE STUDY
}

\author{
SRAVANI KOMMAREDDY ${ }^{1}$, PENTAKOTA KIRANMAYI ${ }^{2 *}$, RAVISUNDER RAGAM ${ }^{2}$, RAMESH BABU K ${ }^{1}$ \\ ${ }^{1}$ Department of Yoga and Consciousness, Andhra University, Visakhapatnam, Andhra Pradesh, India. ${ }^{2}$ Department of Physiology, \\ GITAM Institute of Medical Sciences and Research, GITAM Deemed to be University, Visakhapatnam, Andhra Pradesh, India. \\ Email: studyyoga4@gmail.com
}

Received: 13 December 2021, Revised and Accepted: 20 January 2022

ABSTRACT

Objective: The present study is based on a novel approach of validated breath-holding technique and efficiency of SpO in the adverse $\mathrm{COVID}_{2}-19$ outcomes and comparison with normal subjects.

Methods: It is a prospective observational study conducted in residential/private nursing colleges, St. Luke's School and College of Nursing and Smt. Vijaya Luke's College of Nursing, Visakhapatnam during the period July 2021. Fifty-three student nurses affected with mild COVID-19, 35 student nurses affected with moderate COVID-19, aged 18-23 years were enrolled after taking thorough history about COVID-19 that is after 2 months of complete recovery. They were classified based on the symptom history in which the subjects without symptoms or mild symptoms were taken as mildly affected, whereas subjects with severe symptoms with mild fluctuations in $\mathrm{SpO}_{2}$ who didn't require hospitalization were classified as moderately affected. The study included 109 normal control cases who are never affected with COVID-19 viral infection. In all the subjects, the oxygen saturation was measured using pulse oxymeter and their Breath holding times were also measured using standard protocols.

Results: The mean value of BHT was significantly reduced from normal $16.7339 \pm 3.4$ to $12.8571 \pm 5.1$ (p<0.05) in moderate cases. When oxygen saturation levels were compared before and after the breath holding in normal, mild and moderate cases the results were significant. However, when the oxygen saturation levels were compared between normal and mild COVID-19 cases the values were insignificant ( $\mathrm{p}=0.4)$ and at the same time when the oxygen saturation levels were compared between normal and moderate COVID-19 cases the values were significant ( $\mathrm{p}=0.0001$ ).

Conclusion: According to the findings, breath-holding does not need greater energy expenditure or cardiac output, and it eliminates walking and the related contamination of bystanders as occurring with pulse oximeter. Breath holding time is a determinant of respiratory capacity, when used as parameter helps in assessing the progression of lung injury, it gives an idea about respiratory fitness especially in this COVID era. Breath holding time and fluctuations in $\mathrm{SpO}_{2}$ when used conjointly we can assess degree of lung damage so that further treatment such as the continuity of medication, practicing of breathing exercises with or without medical treatment can be planned. This simple non-invasive tool can be used for the self-assessment of improvement in post-Covid patients. Future validation studies validate this hypothesis, measurement of these basic, innovative surrogates requires minimum inventory (i.e., a means to record oximetry and a timing device) and could feasibly provide a useful way to evaluate risks of future deterioration under under-resourced conditions.

Keywords: Oxygen saturation, Breath holding time, Mild, Moderate, COVID-19.

(C) 2022 The Authors. Published by Innovare Academic Sciences Pvt Ltd. This is an open access article under the CC BY license (http://creativecommons.org/ licenses/by/4.0/) DOI: http://dx.doi.org/10.22159/ajpcr.2022v15i2.43885. Journal homepage: https://innovareacademics.in/journals/index.php/ajpcr

\section{INTRODUCTION}

The current SARS-CoV-2/COVID-19 outbreak has proven as a stark reminder of how novel infections can emerge and spread quickly throughout the human population, posing major public health risks [1]. COVID-19 symptoms include fever, cough, expectoration, headache, breathlessness, myalgia, chest pain, thromboembolic disorders, arthralgia, gastrointestinal disturbances, and metabolic disorders weakness [2]. Asymptomatic and atypical clinical signs have been documented, adding to the complexity of disease transmission dynamics. The infection, on the other hand, can cause pneumonia, multiorgan failure, severe acute respiratory syndrome, and even death in severe cases [2]. In extreme cases, the lung parenchyma is destroyed, resulting in extensive alveolar injury, interstitial thickening, hypoxic vasoconstriction, and ventilation perfusion mismatch, all of which obstruct the vital process of breathing [3]. When compared to children and younger healthy adults, people over the age of 60 and those with chronic health problems such as diabetes, low baseline pulse oxygen saturation $\left(\mathrm{SpO}_{2}\right)$, hypertension, chronic kidney failure, cardiovascular comorbidities, steroid users, and others are more vulnerable to COVID-19 [4]. Despite significant improvement, it is still unclear why some COVID-19 patients have negative outcomes while others recover on their own. Predisposition to hypoxemia or the surprising absence of dyspnea ("silent hypoxemia") in some patients who develop respiratory failure may provide clues [5]. Lower baseline $\mathrm{SpO}_{2}$, a reflection of disease-related gas exchange deficits (e.g., ventilation/perfusion $[\mathrm{V} / \mathrm{Q}]$ heterogeneity), is one of the most consistent risk variables for COVID-19 poor outcomes. In those with early gas exchange deficits (V/Q heterogeneity and lower functional lung gas volumes beyond baseline $\mathrm{SpO}_{2}$, breath holding is likely to result in fast desaturation. This is a quick, non-invasive method of measuring oxygenation that also has the advantage of being continuous, allowing for the detection of unexpected changes in a patient's clinical status. Low blood oxygen, often known as hypoxia, is defined as a measured oxygen saturation of $<94 \%$ in the absence (or $<88 \%$ in the presence) of chronic lung illness. The initial condition progresses insidiously in most people who die of acute COVID-19, often with "silent hypoxia" (hypoxia without clinically discernible symptoms of dyspnea, progressing to pneumonia and acute respiratory distress syndrome, generally in week 2) [5]. The underlying pathophysiology in COVID-19-related hypoxia is most likely a ventilation-perfusion mismatch, which is characterized by a combination of intrapulmonary shunting, loss of lung perfusion control, intravascular microthrombi, and decreased lung compliance, all of which lead to alveolar collapse. 
BHT is a simple evaluation test in which the person is requested to hold their breath as long as they can till they reach their limit following inspiration. It is a determinant of respiratory capacity of an individual which can be used as a tool in the assessment of respiratory fitness. The length of time spent holding your breath is measured in seconds. The point at which breathing can no longer be voluntarily inhibited is called the Breaking point. Due to its favorable connection with spirometric indices, it is commonly employed as a measure of pulmonary function. It is reliant on various variables such as lung volumes, respiratory muscle functioning, illness states, and training level.

The present study is based on a novel approach of validated breathholding technique and efficiency of $\mathrm{SpO}_{2}$ among the normal subjects and COVID affected individuals.

\section{METHODS}

It is a prospective observational study conducted in residential nursing colleges, Visakhapatnam during the period of July 2021. Fifty-three nursing students of mild post COVID-19 cases, 35 cases of moderate Post COVID-19 nursing students aged 18-23 years were enrolled after taking thorough history about COVID-19 that is after 2 months of complete recovery. They were classified based on the symptom history in which the subjects without symptoms or mild symptoms were taken as mildly affected, whereas subjects with severe symptoms with mild fluctuations in $\mathrm{Spo}_{2}$ who dint require hospitalization were classified as moderately affected. The study included 109 normal control cases who are never affected with COVID-19 viral infection. Diagnosis was confirmed with a previous positive nasal or pharyngeal swab or with clear clinical evidence (i.e., typical signs at laboratory blood tests and computed tomography and/or chest ultrasound) when the swab result was yet not available. The previous swab positive reports were checked in all subjects. All the students were given perceived stress scale questionnaire and the students with scores between 0 and 13 which is considered as low stress were enrolled in our study in order to reduce the factor of stress and anxiety which interferes with the results.

\section{Measurement of BHT}

The patient was made to sit up straight with no back support. After that, the patient was told to breathe in at tidal volume and hold the breath at the end of inspiration. The timer was set to keep track of how long each breath was held. The test was continued until the patient required to exhale or the respiratory muscles deviated in any way. Three readings were obtained, with the best of the three being used in the final analysis and being taken as the mean value.

\section{Measurement of $\mathrm{SpO}_{2}$}

Pulse oximeters were used to measure $\mathrm{SpO}_{2}$. The accuracy and reliability of finger pulse oximeters are generally high (provided they have evidence of a quality standard such as ISO 80601-2-61:2017). Pulse oximeter readings should be taken with a warm finger, with the patient sitting erect and relaxing. Allow a minute for the instrument to stabilize before validating the reading.

\section{Measurement of body mass index (BMI)}

BMI is a person's weight in kilograms divided by the square of height in meters.

The triplicate data reading values of $\mathrm{BHT}$ and $\mathrm{Spo}_{2}$ will be analyzed using Statistical Packages for the Social Sciences software, Window version 20.0. Each experimental value will be expressed in terms of mean (SD) (mean and standard deviation). Significance in the difference between the two groups normal with mild and normal with severe will be tested by student t-test, assessed by comparing the corresponding p-value of the test. $\mathrm{p}<0.05$ will be considered significant for the study.

\section{RESULTS}

From Table 1, it was observed that the mean value of BHT was significantly reduced from normal $16.7339 \pm 3.4$ to moderate cases12.8571 $\pm 5.1(\mathrm{p}<0.05)$ but insignificant findings of BHT $(\mathrm{p}>0.05)$ were noticed when the mean values were compared between normal $16.7339 \pm 3.4$ and mild cases $15.5849 \pm 4.3$. When oxygen saturation levels were compared before and after the breath holding in normal, mild, and moderate cases the results were significant (Table 2). However, when the oxygen saturation levels were compared between normal and mild COVID-19 cases the values were insignificant $(p=0.4)$ and at the same time when the oxygen saturation levels were compared between normal and moderate COVID-19 cases the values were significant $(\mathrm{p}=0.0001)$ (Table 3). The mean value of body mass index was similar $(21.6 \pm 3.8,21.8 \pm 4.0)$ in normal and mild COVID-19 cases but somewhat lesser mean value in moderate cases $(20.7 \pm 3.9)$. However, from the results, it was noticed that, $\mathrm{BMI}$ is an independent and insignificant variable in the present study ( $p>0.05)$ (Table 4$)$. ). All the 3 variables BHT, Spo 2 after breath holding and BMI of normal, mild and moderate cases are compared in normal,mild and moderate case (Fig. 1). It clearly indicates a lower BHT in moderate cases compared to mild and normal cases. The differences in the BMI is not very significant,as this study enrolled subjects who fall under normal BMI.

\section{DISCUSSION}

Breath holding time and oxygen saturation levels $\mathrm{SpO}_{2}$ are becoming significant variables in the outcome of COVID-19 cases, according to the findings of this study, and are more prominent characteristics in the COVID-19 literature. These findings, which come from 109 normal COVID-19 cases and 35 moderate COVID-19 cases, demonstrate that $\mathrm{SpO}_{2}$ readings from pulse oximetry had slightly lower levels of agreement in the moderate symptoms patient group, but that it is still a useful clinical diagnostic tool. The $\mathrm{SpO}_{2}$ was determined using a pulse oximeter in this investigation. Pulse oximeters are painless and noninvasive devices that detect blood oxygen saturation levels. The use of pulse oximetry devices in COVID-19 pandemic investigations is becoming more valuable. The use of oximeters in low-resource settings and forecasting clinical deterioration are among the research topics.

One study found that breath holding creates hypoxic situation in the body which has lot of benefits. Multiple factors, such as mobility, perfusion, and skin pigmentation, can affect the accuracy of pulse oximetry [6]. The rise in arterial $\mathrm{pCo}_{2}$ and fall in $\mathrm{pO}_{2}$ stimulate central and peripheral chemoreceptors, which stimulate respiration which is taken as Break point of BHT. Generally breaking point is reached at alveolar $\mathrm{pO}_{2}$ of $56 \mathrm{~mm} \mathrm{Hg}$ and alveolar $\mathrm{pCo}_{2}$ of $49 \mathrm{~mm}$ of $\mathrm{Hg}$. Thus, breath holding decreases the oxygen levels in the body producing Hypoxia which stimulates the respiratory centers to continue breathing. Hameed et al. found that the production of reactive oxygen species (ROS) increased in ependymoglia stem cells after hypoxia and re-oxygenation. The ROS were produced by the mitochondria in the cells, and by NADPH oxidase complex (NOX) proteins in the cell membrane. Experiments with drugs that inhibit the production of ROS showed that only the ROS that were produced by the NOX proteins are required for the ependymoglia stem cells to increase in number [7]. Thus hypoxia has a protective effect by stimulating various repairing

Table 1: Effect of breath holding time in normal, mild, and moderate cases of COVID-19

\begin{tabular}{llll}
\hline Parameter BHT & Normal & Mild & Moderate \\
\hline Mean & 16.7339 & 15.5849 & 12.8571 \\
Median & 17.0000 & 15.0000 & 14.0000 \\
SD & 3.4498 & 4.3828 & 5.1114 \\
SE & 0.3304 & 0.6020 & 0.8640 \\
$95 \%$ CONF & 0.6550 & 1.2081 & 1.7558 \\
$99 \%$ CONF & 0.8665 & 1.6098 & 2.3574 \\
Size & 109.0000 & 53.0000 & 35.0000 \\
& & "t" value: 1.233 & "t" value: 3.44 \\
& & p value: 0.22 & p value: 0.001 \\
& & (Insignificant) & (Significant) \\
\hline
\end{tabular}

SD: Standard deviation 
Table 2: Effect of oxygen saturation in normal, mild and moderate cases of COVID-19

\begin{tabular}{|c|c|c|c|c|c|c|}
\hline \multirow[t]{2}{*}{ Parameter $\mathrm{Spo}_{2}$} & \multicolumn{2}{|l|}{ Normal } & \multicolumn{2}{|l|}{ Mild } & \multicolumn{2}{|c|}{ Moderate } \\
\hline & Before & After & Before & After & Before & After \\
\hline Mean & 98.1927 & 96.3670 & 98.0755 & 95.8491 & 95.8000 & 95.0000 \\
\hline SD & 1.6749 & 2.4406 & 1.3847 & 3.0659 & 2.6324 & 2.9803 \\
\hline SE & 0.1604 & 0.2338 & 0.1902 & 0.4211 & 0.4450 & 0.5038 \\
\hline $95 \%$ CONF & 0.3180 & 0.4634 & 0.3817 & 0.8451 & 0.9043 & 1.0238 \\
\hline $99 \%$ CONF & 0.4207 & 0.6130 & 0.5086 & 1.1261 & 1.2141 & 1.3746 \\
\hline Size & 109.0000 & 109.0000 & 53.0000 & 53.0000 & 35.0000 & 35.0000 \\
\hline
\end{tabular}

SD: Standard deviation

Table 3: Comparison of Oxygen saturation after breath holding of normal cases with mild and moderate cases of COVID-19

\begin{tabular}{ll}
\hline Parameter & “p”value \\
Spo $_{2}$ & \\
\hline Normal & \\
$\quad 96.3670 \pm 2.4$ & \\
Mild & \\
$\quad 95.8491 \pm 3.06$ & $\mathrm{p}=0.4$ \\
Moderate & \\
$\quad 95.0000 \pm 2.9$ & $(\mathrm{p}=0.0001)$ \\
\hline
\end{tabular}

Table 4: Effect of BMI in normal, mild, and moderate cases of COVID-19

\begin{tabular}{llll}
\hline $\begin{array}{l}\text { Parameter } \\
\text { BMI }\end{array}$ & Normal & Mild & Moderate \\
\hline Mean & 21.6156 & 21.8585 & 20.7886 \\
Median & 21.5000 & 22.0000 & 19.8000 \\
SD & 3.8458 & 4.0017 & 3.9171 \\
SE & 0.3684 & 0.5497 & 0.6621 \\
$95 \%$ CONF & 0.7302 & 1.1030 & 1.3456 \\
$99 \%$ CONF & 0.9660 & 1.4698 & 1.8066 \\
Size & 109.0000 & 53.0000 & 35.0000 \\
& & "t" value: -1.9709 & "t" value: 0.70 \\
& & p value: 0.05 & p value: 0.48 \\
& & (Insignificant) & (In Significant) \\
\hline
\end{tabular}

BMI: Body mass index, SD: Standard deviation

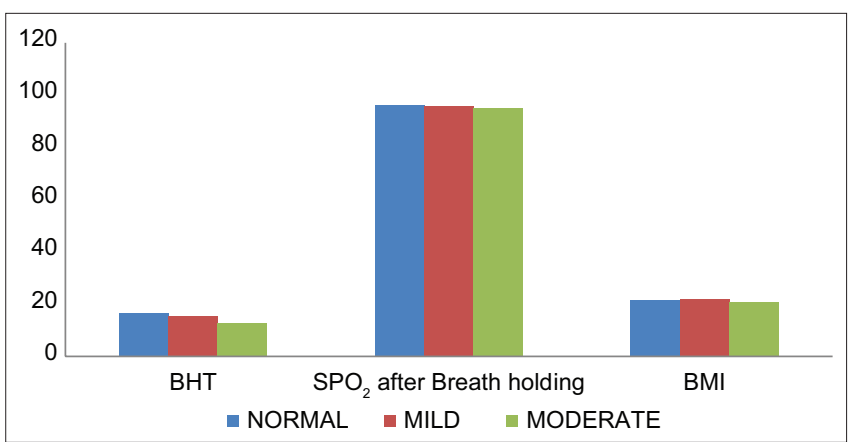

Fig. 1: Impact of $\mathrm{BHT}, \mathrm{SPO}_{2}$, and Body mass index in COVID-19

mechanisms. Practicing of BHT as a part of breathing exercises increases respiratory capacity in moderately affected COVID patients and it also reduces anxiety levels related to stress. A larger disparity was discovered between oxygen saturation $\left(\mathrm{SpO}_{2}\right)$ and respiratory rates in COVID-19 Acute Respiratory Failure (ARF) patients compared to previous non-COVID-19 ARF patients in a research conducted by Jouffroy et al. (2020) from prehospital first responder data. Normal breathing rates could hide significant hypoxia without a
$\mathrm{SpO}_{2}$ measurement, making severity assessment more challenging in an out-of-hospital context [6]. The BHT values were significantly lowered from normal to moderate instances in the study, however there were no significant findings when the values were compared between normal and mild cases. According to the findings, breathholding does not need greater energy expenditure or cardiac output, and it eliminates walking and the related contamination of bystanders and caregivers. Should future validation studies support this concept, measurement of these simple, novel surrogates capturing pulmonary and chemoreflex risk factors requires minimal inventory (i.e., a means to record oximetry and a timing device) and could feasibly provide a useful way to estimate risks of future deterioration in underresourced circumstances.

\section{CONCLUSION}

According to the findings, breath-holding does not need greater energy expenditure or cardiac output, and it eliminates walking and the related contamination of bystanders as occurring with pulse oximeter. Breath holding time is a determinant of Respiratory capacity, when used as parameter helps in assessing the progression of lung injury; it gives an idea about respiratory fitness especially in this COVID era. Breath holding time and fluctuations in $\mathrm{Spo}_{2}$ when used conjointly we can assess degree of lung damage so that further treatment like the continuity of medication, practicing of breathing exercises with or without medical treatment can be planned. This simple non-invasive tool can be used for the self-assessment of improvement in Post-COVID patients. Practicing of BHT as a part of breathing exercises increases respiratory capacity in moderately affected COVID patients and it also reduces anxiety levels related to stress. Future validation studies validate this hypothesis, measurement of these basic, innovative surrogates requires minimum inventory (i.e., a means to record oximetry and a timing device) and could feasibly provide a useful way to evaluate risks of future deterioration under under-resourced conditions.

\section{AUTHOR CONTRIBUTIONS}

The first author of the article, SK had performed the work, collected the literature and performed the statistical analysis. The corresponding author KP wrote the first draft of the manuscript. The third and fourth author RR and KR corrected the manuscript.

\section{CONFLICT OF INTEREST}

The authors declared no conflict of interest.

\section{SOURCE OF FUNDING}

The study was not supported by any grants and funds.

\section{REFERENCES}

1. Zheng J. SARS-CoV-2: An emerging Coronavirus that causes a global threat. Int J Biol Sci 2020;16:1678-85.

2. Raveendran AV, Jayadevan R, Sashidharan S. Long COVID: An 
overview. Diabetes Metab Syndr 2021;15:869-75.

3. Carotti M, Salaffi F, Sarzi-Puttini P, Agostini A, Borgheresi A, Minorati D, et al. Chest CT features of Coronavirus disease 2019 (COVID-19) pneumonia: Key points for radiologists. Radiol Med 2020;125:636-46

4. Mejía F, Medina C, Cornejo E, Morello E, Vásquez S, Alave J, et al. Oxygen saturation as a predictor of mortality in hospitalized adult patients with COVID-19 in a public hospital in Lima, Peru. PLoS One 2020;15:e244171
5. Messineo L, Perger E, Corda L, Joosten SA, Fanfulla F, Pedroni L, et al. Breath-holding as a novel approach to risk stratification in COVID-19. Crit Care 2021;25:208.

6. Jouffroy R, Jost D, Prunet B. Prehospital pulse oximetry: A red flag for early detection of silent hypoxia in COVID-19 patients. Crit Care 2020;24:313.

7. Hameed LS, Berg DA, Belnoue L, Jensen LD, Cao Y, Simon A. Environmental changes in oxygen tension reveal ROS-dependent neurogenesis and regeneration in the adult newt brain. eLife 2015;4:08422. 